\title{
A rare case of pleomorphic adenoma with difficult diagnosis using biopsy
}

\author{
Akifumi Nakamura ${ }^{1}$, Teruhisa Takuwa ${ }^{1}$, Nobuyuki Kondo ${ }^{1}$, Takahiro Watanabe ${ }^{2}$, Seiichi Hirota ${ }^{2}$, Seiki \\ Hasegawa $^{1}$
}

${ }^{1}$ Department of Thoracic Surgery, ${ }^{2}$ Department of Surgical Pathology, Hyogo College of Medicine, Nishinomiya, Hyogo, Japan Correspondence to: Akifumi Nakamura, MD. Department of Thoracic Surgery, Hyogo College of Medicine, 1-1 Mukogawa-cho, Nishinomiya, Hyogo 663-8501, Japan. Email: xxmp97511@zeus.eonet.ne.jp.

\begin{abstract}
An 85-year-old woman was found to have a nodule in the left S6 of the lung on computed tomography (CT); the pathological diagnosis from the CT-guided biopsy was adenoid cystic carcinoma. We accordingly performed left lower lobectomy. Pathologically, the biphasic cell population showed admixtures in varying proportions of epithelial and stromal elements, thereby indicating pleomorphic adenoma (PA). PA of the lung is extremely rare with reports of only 20 patients so far. Interestingly, this case was difficult to diagnose using CT-guided biopsy because the overview and biphasic cell population obtained on biopsy were garbled. Here we have reported our rare experience of PA.
\end{abstract}

Keywords: Pleomorphic adenoma (PA); biopsy; diagnosis

Submitted Apr 13, 2018. Accepted for publication Jul 13, 2018.

doi: $10.21037 /$ jtd.2018.07.82

View this article at: http://dx.doi.org/10.21037/jtd.2018.07.82

\section{Introduction}

Pleomorphic adenoma $(\mathrm{PA})$ is the most common benign neoplasm of the salivary glands of the head and neck and consists of epithelial cells and modified myoepithelial cells intermingled with myxoid or chondroid stroma. PA of the lung is extremely rare and difficult to diagnose (1). In the present case, the diagnosis of PA was difficult using computed tomography (CT)-guided biopsy because the overview and biphasic cell population obtained were garbled. Finally, we confirmed the overview of the tumor from a surgical specimen to diagnose PA. To the best of our knowledge, to date, no clinical reports have described the differences between the characteristics of a biopsy specimen and a surgical specimen for PA. We have reported here an extremely rare case of PA.

\section{Case presentation}

An 85-year-old woman diagnosed with carcinoma of the ampulla of Vater was referred to our hospital. Her preoperative CT examination revealed a solid nodule with a $19-\mathrm{mm}$ maximum diameter in the left $\mathrm{S} 6$ of the lung (Figure 1A). Positron-emission tomography/CT revealed an accumulation of $18 \mathrm{~F}$-fluorodeoxyglucose with a maximum standardized uptake value of 2.96 (Figure 1B). No lymph-node metastases or distant metastases were detected. The patient's vital capacity was $1.73 \mathrm{~L}$ (93.0\% predicted), and her forced expiratory volume in 1 second $\left(\mathrm{FEV}_{1}\right)$ was $1.26 \mathrm{~L}$ (68.1\% $\left.\mathrm{FEV}_{1} \%\right)$. A CT-guided percutaneous needle biopsy of the pulmonary nodule revealed (Figure 2A,B) oval tumor cells arranged in tubes, cribriform, and solid patterns, which are the morphological characteristics of adenoid cystic carcinoma (ACC) (c-T1bN0M0, c-stage IA2). On the basis of these results, the patient was referred to our department for further examination and treatment. Our cancer board therefore contemplated the most suitable approach. Carcinoma of the ampulla of Vater is a more aggressive tumor with poor prognosis compared with ACC and a profound association reported with obstructive jaundice. We decided to perform pancreatic duodenectomy for the carcinoma of the ampulla of Vater because of the abovementioned factors. Thereafter, the patient followed 

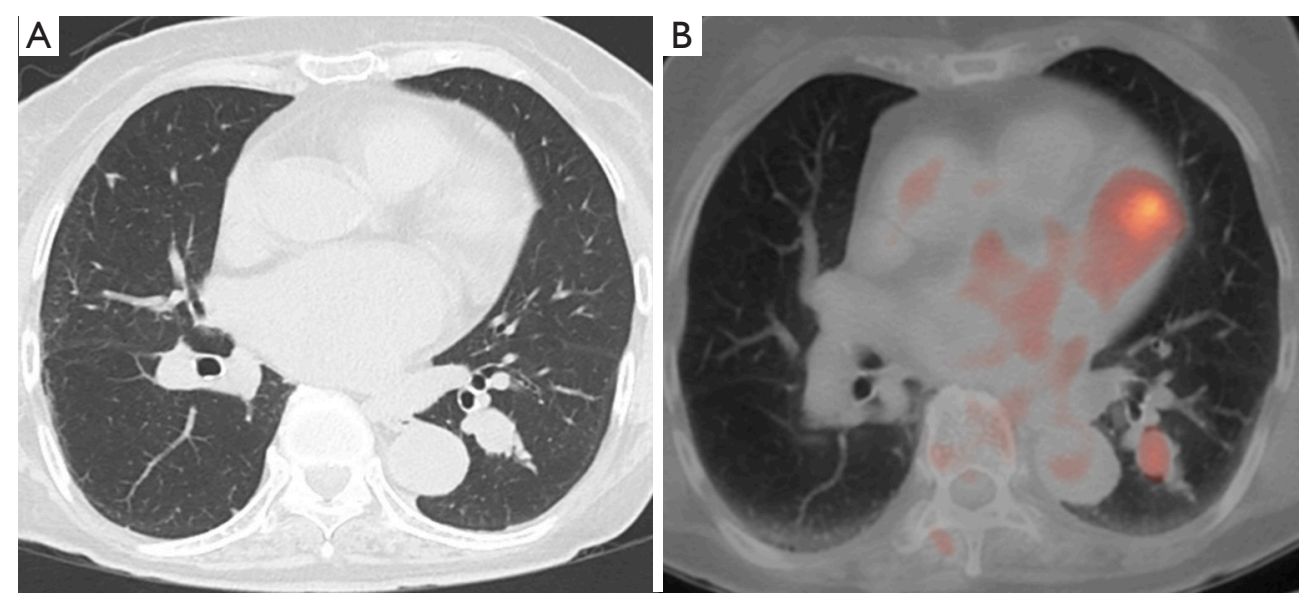

Figure 1 Preoperative CT and FDG-PET/CT. (A) CT revealing a 19-mm solid nodule in the left lower lobe. (B) FDG-PET/CT revealing accumulation of 18F-FDG with an SUVmax of 2.96. CT, computed tomography; FDG-PET/CT, positron-emission tomography with 2-deoxy-2-[fluorine-18]-fluoro-D-glucose integrated with computed tomography.
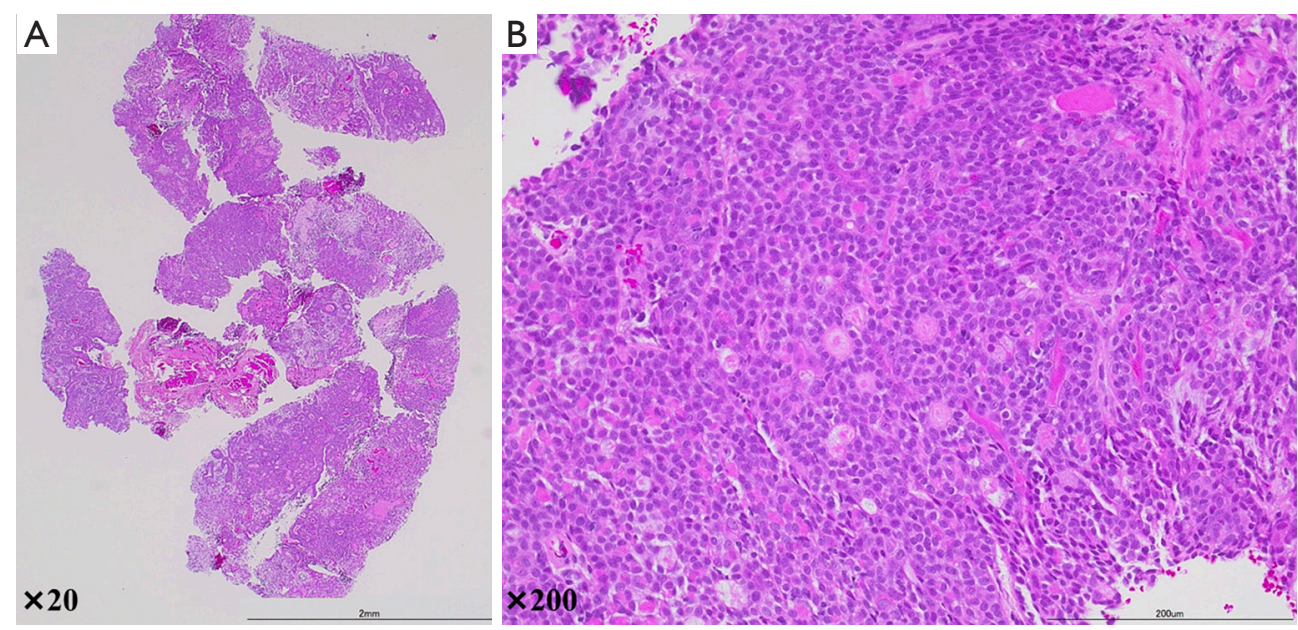

Figure 2 Microscopic pathology of a CT-guided biopsy. A CT-guided biopsy specimen of the tumor consisting of oval tumor cells, arranged in solid and cribriform patterns $(\mathrm{H} \& \mathrm{E}),(\mathrm{A}) \times 20,(\mathrm{~B}) \times 200$. CT, computed tomography; H\&E, hematoxylin and eosin staining.

an uneventful course with no complications.

Next, we performed a pulmonary operation with minimally invasive open surgery, which was video-assisted by small posterolateral thoracotomy (7-cm incision). The tumor was located in the left $\mathrm{S} 6$ region. Thus, we performed the left lower lobectomy and systematic lymph-node dissection. The operating time was 119 min with a blood loss of $10 \mathrm{~mL}$. The postoperative course was uneventful. We removed the chest drain 2 days after the surgery.

Overall, the tumors were well-demarcated and lobulated with a fleshy-to-firm whitish gray cut surface. Pathologically, the microscopic findings revealed that the tumor displayed different growth patterns, a biphasic arrangement including a glandular or tubular structure, solid cell nests, and myxoid or chondroid areas. The neoplastic epithelial cells included myoepithelial cells and glandular epithelial cells (Figure 3A,B,C). Mild cellular atypia and no foci of necrosis or mitoses were found. Taken together, the patient was diagnosed with primary PA. The patient followed-up for 1 year since the operation without 

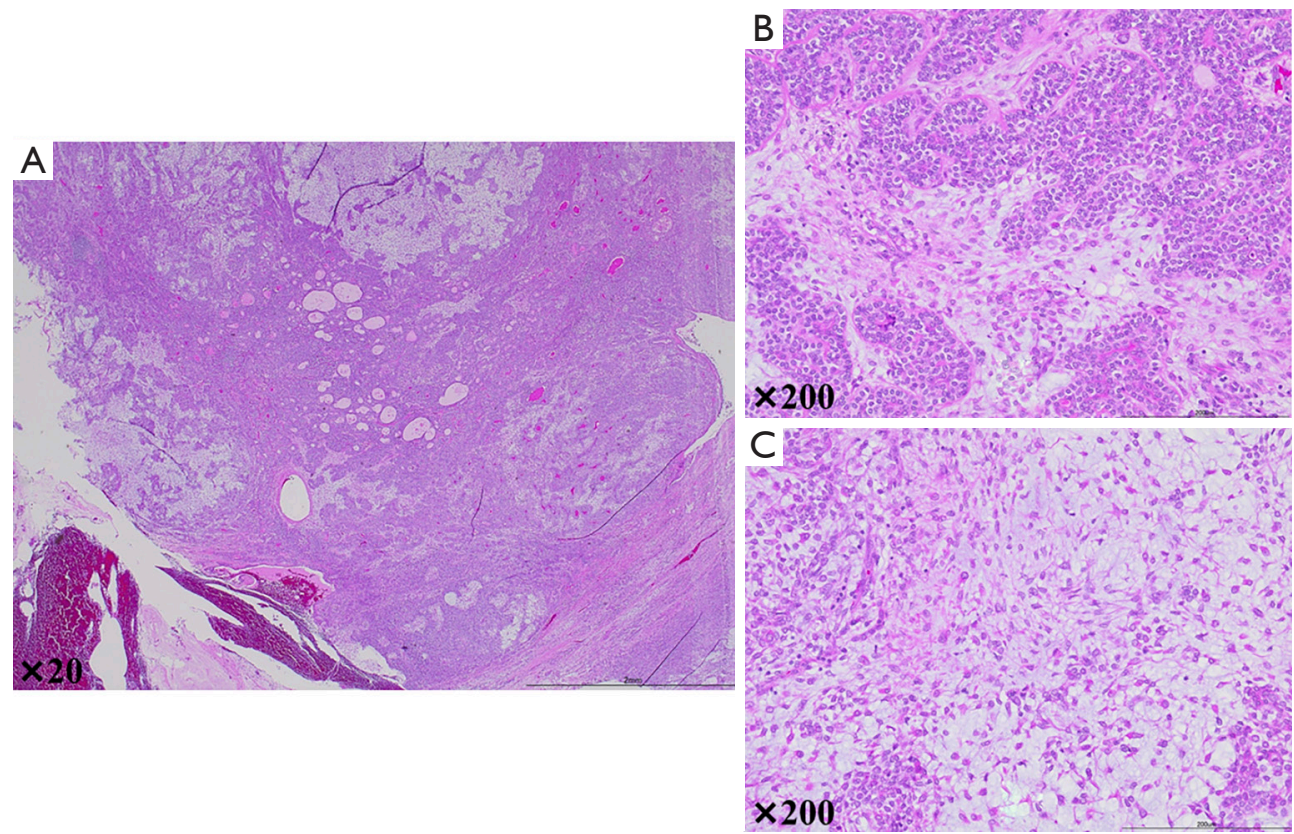

Figure 3 Microscopic pathology of the surgical specimen. (A) A surgical specimen of the tumor showing different growth patterns, a biphasic cell population, with epithelial and stromal components (H\&E, $\times 20)$. (B) The neoplastic epithelial cells including myoepithelial cells and glandular epithelial cells $(\mathrm{H} \& \mathrm{E}, \times 200)$. (C) Chondromyxoid stroma (H\&E, $\times 200)$. H\&E, hematoxylin and eosin staining.

any recurrence or need for any further treatment.

\section{Comments}

PA of the lung is extremely rare, and to the best of our knowledge, there are only 20 cases reported so far in the English literature (1). PA is classified as a benign salivary gland-type tumor according to the current World Health Organization classification of lung tumors. Most tumors are associated with the trachea and major or secondary bronchus. Typically, on bronchoscopy, the tumor exhibits a well-demarcated and polypoid mass. The histological features of PA of the lung are identical with those of lesions arising in the salivary gland. PA comprises a biphasic cell population with varying proportions of epithelial cells and modified myoepithelial cells intermingled with myxoid or chondroid stroma that have prominent glandular or ductal components embedded in the chondromyxoid stroma. Solid islands of epithelial cells with a focal myxoid stroma occur in such tumors (2).

In this context, varied pathological findings have been reported for PA, making it difficult to diagnose using a biopsy specimen. In our patient, interestingly, the overview and biphasic cell population obtained from the CT-guided biopsy were garbled because only a part of the tumor was gathered. However, the surgical specimen exhibited a biphasic cell population with varying proportions of epithelial cells and modified myoepithelial cells intermingled with myxoid or chondroid stroma. Finally, based on the cumulative findings, the patient was diagnosed with PA of the lung. Thus, we can frequently only confirm the diagnosis of a portion of the tumor appearing from the biopsy specimen without proving the biphasic cell population. Due to this, there are many cases of PA that are difficult to diagnose using a biopsy specimen.

Importantly, PA may possibly undergo malignant transformation, metastases, or recurrence, as has been reported in $10-22 \%$ of patients with PA (2). Moran et al. (3) reported that tumors with malignant features in components such as prominent necrosis, cellular atypia, $\geq 5$ mitoses per $2 \mathrm{~mm}^{2}$, and vascular invasion are predisposed to develop recurrent and metastatic PA. For reference, this case did not exhibit mitoses or prominent necrosis but revealed a mild cellular atypia. Furthermore, Moran et al. described that PA with these components should be called carcinoma ex PA, malignant mixed tumor (3). In contrast, Tokunaga et al. (4) 
reported a case of recurrence as pleural dissemination after percutaneous CT-guided needle biopsy, followed by the complete resection of PA. Tokunaga et al. stated that there is a possibility that the local failure is associated with percutaneous needle biopsy, and as such, warrants attention.

Owing to its rarity, the best treatment options for PA of the lung remain to be established. Most of the past reports described surgical resection for PA. Furthermore, some reports described the local recurrence and metastasis of PA after surgery, whereas others reported the difficulty in diagnosing PA using biopsy specimen or intraoperative frozen section. Therefore, we recommend performing lobectomy in proportion to the malignant tumor if the patients have a good general condition and normal lung function.

We have reported an extremely rare case of PA. The pathological findings of the disease were different between CT-guided biopsy specimen and surgical specimen, and this made the diagnosis difficult. Considering that PA can occasionally develop recurrence and metastases after surgery, we highly recommend that surgical treatment and postoperative surveillance be performed in proportion to the malignant tumor.

\section{Acknowledgements}

We thank S Matsumoto, A Kuroda, T Nakamichi, A Fukuda for their comments on the manuscript.

Cite this article as: Nakamura A, Takuwa T, Kondo N, Watanabe T, Hirota S, Hasegawa S. A rare case of pleomorphic adenoma with difficult diagnosis using biopsy. J Thorac Dis 2018;10(8):E630-E633. doi: 10.21037/jtd.2018.07.82

\section{Footnote}

Conflicts of Interest: The authors have no conflicts of interest to declare.

Informed Consent: Witten informed consent was obtained from the patient for the writing of this manuscript.

\section{References}

1. Wang Y, Lei L, Jiang GY, et al. Primary pleomorphic adenoma of the lung with positive staining of TTF-1: a case report and review of literature. Int J Clin Exp Med 2018;11:378-83.

2. Travis WD, Brambilla E, Burke AP, et al. WHO classification of tumours of the lung, pleura, thymus and heart. Lyon: International Agency for Research on Cancer, 2015.

3. Moran CA, Suster S, Askin FB, et al. Benign and malignant salivary gland-type mixed tumors of the lung. Clinicopathologic and immunohistochemical study of eight cases. Cancer 1994;73:2481-90.

4. Tokunaga T, Kunou H, Ishida D, et al. Pleural recurrence after surgery for pleomorphic adenoma arising from peripheral lung: computed tomographyguided percutaneous needle biopsy as a possible mechanism of local failure. Gen Thorac Cardiovasc Surg 2015;63:478-82. 